\title{
Source Location of Forced Oscillations Using Synchrophasor and SCADA Data
}

\author{
James G O’Brien \\ Pacific Northwest National \\ Laboratory \\ Richland WA \\ james.obrien@pnnl.gov
}

\author{
Tianying $\mathrm{Wu}$ \\ Washington State University \\ Pullman WA \\ tianying.wu@wsu.edu
}

\author{
Vaithianathan "Mani" \\ Venkatasubramanian \\ Washington State University \\ Pullman WA \\ mani@eecs.wsu.edu
}

\author{
Hongming Zhang \\ Peak Reliability \\ Loveland CO \\ hzhang@peakrc.com
}

\begin{abstract}
$^{1}$
Recent advances in synchrophasor based oscillation monitoring algorithms have allowed engineers to detect oscillation issues that may have previously gone undetected. Although such an oscillation can be flagged and its oscillation shape can indicate the general vicinity of its source, low number of synchrophasors means that a specific generator or load that is the root cause of an oscillation cannot easily be pinpointed. Fortunately, SCADA serves as a much more readily available telemetered source of data if only at a relatively low sampling rate of 1 sample every 1 to 10 seconds. This paper shows that it is possible to combine synchrophasor and SCADA data for effective source location of forced oscillations. For multiple recent oscillation events, the proposed automatic methods were successful in correct identification of the oscillation source which was confirmed in each case by discussion with respective generation plant owners.
\end{abstract}

\section{Introduction}

Different from the natural electromechanical modal oscillations, forced oscillations in power systems are caused by external sources such as cyclic loads and control failures in generator sites [1],[2]. The identification of these forced oscillations has been a significant problem for the industry. Because of the increase in PMU visibility, there is significant work being done in detecting and locating forced oscillations using synchrophasor measurements [3]-[6]. The source location becomes difficult especially when there is resonance between the forced oscillation and a system

\footnotetext{
${ }^{1}$ The author James O'Brien was with Peak Reliability while doing the research reported in this paper.
}

inter-area mode [6] which will not be discussed in this paper.

However, the observability of oscillation sources using PMU data is less than ideal. Even though many hundreds of PMUs have been installed across the power grid in North America, most of these are located for monitoring transmission corridors and do not provide much coverage of generation facilities. Therefore, the indication from the oscillation shapes produced by oscillation detection software that uses PMU data is not specific enough for source location of problematic oscillations. In our experience, oscillation shape from PMU data analysis typically points towards a portion of the system with dozens of candidate generation sites. With low PMU visibility, source location of forced oscillations becomes difficult to produce tangible results without exhaustive manual analysis.

Typically, other sources of data need to be examined for their use in this application. The most readily available source is the SCADA data that is received throughout the western interconnection (commonly referred to as Western Electricity Coordinating Council (WECC) power system) by Peak Reliability. Currently while we only have PMU visibility of tens of generators in the WECC system, the SCADA visibility covers more than 2000 generators. Both real and reactive power outputs are monitored from each generator.

Due to the low sampling rate of SCADA data and its non-synchronized nature, it is a technical challenge to detect forced oscillations using SCADA data. However, once a possible forced oscillation has been detected with PMU measurements, SCADA data can then be used for locating the specific source. As an example, the plot below shows the analog frequency chart recording of an oscillation event in 1992 [7] that lasted for about 38 minutes. Note that the difference between normal ambient conditions versus sustained oscillations is clearly observable in Figure 1 even 
though the $1 \mathrm{~Hz}$ oscillations were much faster than the slow time response of the analog recorder.

The challenge then becomes how to properly identify from the multitude of SCADA data such distinctly different responses between when the oscillations of interest are present versus when they are not. This paper proposes two strategies for automatically selecting and ranking likely generator oscillation sources from their recorded SCADA measurements. Synchrophasor based oscillation monitoring is used first to estimate the onset of a forced oscillation so that the distinction between ambient and oscillation conditions can be made. Oscillation shape of the forced oscillation from synchrophasors can also be used to validate the results of SCADA based ranking in most cases excepting when inter-area resonance is in effect [6].

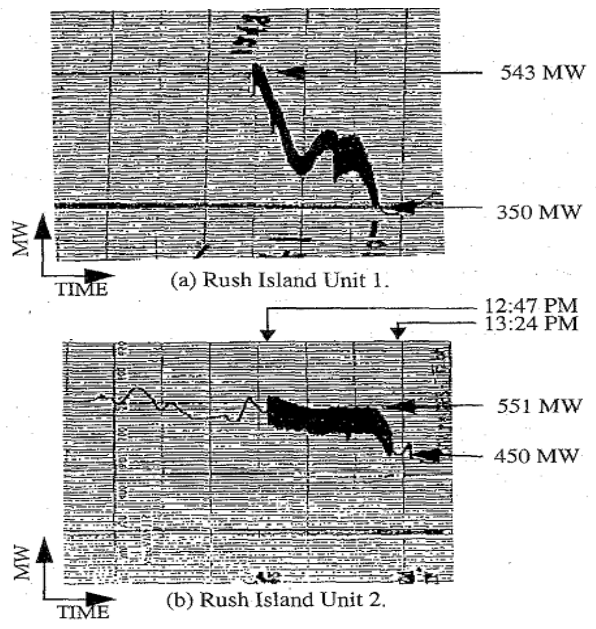

Figure 1. MW chart record of the Rush Island event on June 12, 1992 [7]

In this paper, two slightly different methods are proposed in order to locate the sources of forced oscillations using SCADA data. One is denoted Pattern Mining Algorithm (PMA), which considers the number of high-amplitude peaks in SCADA data during the time periods when oscillations were detected by the PMU engines as the key factor in ranking. The second one is called Maximal Variance Ratio Algorithm (MVRA) which ranks the SCADA signals based on the ratio of the average variances during oscillation and ambient time periods. The two methods serve to crosscheck the ranking with each other.

The rest of the paper is organized as follows: Section 2 introduces a recent oscillation event in western interconnection power system, which motivated this paper. The two methods of locating the sources of forced oscillations using SCADA data are then proposed in Section 3, and are illustrated on the event in Section 2. In Section 4, two other oscillation events are studied. Section 5 concludes the paper.

\section{Event 1 on January 27, 2015}

A recent oscillation event (denoted Event 1) occurred on January 27, 2015 in western interconnection power system. It has been detected during offline studies using Fast Frequency Domain Decomposition (FFDD) algorithm [8].

As shown in Figure 2(a), the oscillation started at approximately 11:00 AM and lasted till about 11:40 AM according to FFDD analysis of the WECC PMU data. Analysis window length for FFDD was 180 seconds which was updated every 10 seconds in a moving window formulation [8]. The oscillation frequency was at around $1.12 \mathrm{~Hz}$ with the damping ratio estimated to be below $1 \%$. Figure 2(b) shows the average oscillation shape for the $1.12 \mathrm{~Hz}$ oscillation of Figure 2(a). PMU signals with the largest oscillation shape magnitudes were all from the same area, with low PMU coverage consisting of more than 50 generation substations. The presence of the forced oscillation can be clearly seen in the line current measurement from one of the PMUs in Figure 2(c). Although the oscillation magnitude is small, the FFDD algorithm [8] can detect it well as shown in the summary plot Figure 2(a).

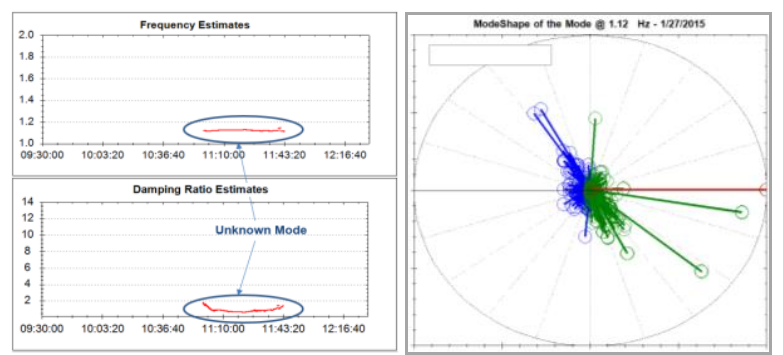

(a) Summary plot (b) Oscillation shape

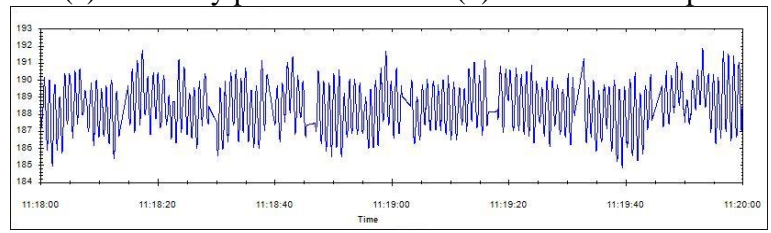

(c) Line current measured by one PMU

Figure 2. Detection of the forced oscillation using FFDD

In order to identify the specific source of the forced oscillation, three-hour SCADA data (9:30 AM to 12:30 PM) recorded from over 2000 generators in western interconnection power system was used for analysis. The discussion in this paper assumes the oscillation source to be a generator while similar analysis can be applied for load sources as well. 
Because of the vast amount of SCADA data, even with the indication from oscillation shape results, it took several weeks to identify the oscillation source through the manual search process. Subsequently, efficient methods have been developed for locating the source of forced oscillations automatically from among thousands of SCADA signals.

\section{Use of SCADA data for source location}

Unlike the high sampling rate PMU data, the SCADA system only provides data from generators and substations every 1 to 10 seconds. Measurement and collection of SCADA data are not timesynchronized. Therefore, SCADA data has been first interpolated like a synchronized stream of data with one sample every 10 seconds. The sampling rate then becomes $0.1 \mathrm{~Hz}$ which suggests that the corresponding Nyquist frequency is $0.05 \mathrm{~Hz}$. It is a challenge to analyze a forced oscillation at $1.12 \mathrm{~Hz}$ of Event 1 with such low sampling rate data using any existing signal processing technique.

Another challenge in using SCADA data is that the signals are recorded with different resolutions (tenth of MW or MVAR for some channels, and one MW or MVAR for some channels). Moreover, the sensitivities of the signals vary a lot in different areas of the system. Therefore, some of the signals can be very noisy, whereas others may remain unchanged for long periods.

Two methods that can handle all these practical issues are proposed in this section. Purely from the very large number of generators in a large power system, it is very difficult to identify the oscillation source effectively while avoiding false alarms. For this purpose, the two slightly different methods can be used to reinforce the ranking of possible source locations.

The start and end time of the forced oscillation need to be known from a PMU based oscillation detection engine such as by using FFDD of [8] before applying these two methods to the SCADA data. The time window in between the start and end times of oscillation detection will be referred to as the oscillation window for the rest of the paper. Nonoscillation time period is accordingly referred to as the ambient window. The oscillation window of Event 1 in Section II is from 11:00 AM to 11:40 AM.

In order to properly distinguish from a generator which shows oscillating characteristics within the oscillation window, versus another generator that has these characteristics throughout the entire data set, an initial ambient window is needed for baselining.
Both methods will compute the ranking index for all the generator outputs, where $\mathrm{n}$ is the number of channels.

\subsection{Pattern Mining Algorithm (PMA)}

To begin with, the data goes through a prescreening process (denoted the data sanity check) to ensure that the generator is in use and there is some minimum variation within the output for the set. The channel whose maximal output is less than $10 \mathrm{MW}$ or MVAR, and the one whose maximal difference for the entire data set is less than $1 \mathrm{MW}$ or MVAR are ignored.

Next, a 25-point median filter is applied for detrending. The absolute values of the differences between the raw measurements and the filtered data, denoted the detrended data, can be used as a measure of the oscillation activity as seen in the SCADA signal. When an oscillation occurs, the amplitude of the differences during the oscillation window should be relatively higher than the amplitudes during the ambient window. Accordingly, a threshold is needed in order to rule out small differences. The threshold (denoted the $3 \sigma$ threshold) is set to be three times the standard deviation of the detrended data in the ambient window.

This method then counts the number of the highamplitude peaks in the raw measurements whose detrended values are outside the $3 \sigma$ threshold in the oscillation window, denoted $N U M_{\text {osc }}$, and in the ambient window, denoted $N U M_{a m b}$. In this context, the amplitude of the peaks is ignored per se. The ranking index of each channel is then formulated as,

$$
K_{P M A_{-} i}=\frac{N U M_{\text {osc }_{-} i}}{\text { Length }_{\text {osc }}}-\frac{N U M_{a m b_{-} i}}{\text { Length }_{a m b}}, \quad i=1,2, \ldots, n,(1)
$$

where Length osc $_{\text {and Length }}$ amb represent the lengths (total number of samples) of the oscillation and the ambient windows, respectively.

In order to determine relative ranking between the generators, the main steps of the pattern mining algorithm are summarized as below.

1) Input SCADA data of generators and the oscillation event time as detected by FFDD using PMU data.

2) Data sanity check.

3) Apply the median filter and subtract the median filtered data from the raw data for detrending.

4) Calculate the absolute values of the differences between the raw measurements and the filtered data.

5) Reject the channel if the maximal absolute value of the differences is less than $1 \mathrm{MW}$ or MVAR.

6) Count $N U M_{o s c_{-} i}$ and $N U M_{a m b} i$.

7) Compute the ranking index $K_{P M A_{-} i}$ based on (1). 
8) Apply step 2 to 7 for the rest of channels.

9) Select Top 3 channels based on the ranking index.

10) Inspect the MW outputs of the possible oscillation sources for manual verification.

Table 1. Possible sources identified using PMA

\begin{tabular}{|c|c|c|c|c|}
\multicolumn{2}{|c}{ Length $_{\text {osc }}=241$, Length $_{\text {amb }}=840}$. \\
\hline Ranking & Channel Name & $\begin{array}{c}\text { Ranking } \\
\text { Index } \boldsymbol{K}_{\boldsymbol{P M A}}\end{array}$ & NUM $_{\text {osc }}$ & $\boldsymbol{N U}_{\boldsymbol{\text { amb }}}$ \\
\hline 1 & Generator 1085 & 0.2264 & 56 & 5 \\
\hline 2 & Generator 1088 & 0.1250 & 37 & 24 \\
\hline 3 & Generator 1087 & 0.1155 & 33 & 18 \\
\hline
\end{tabular}

Event 1 from Section 2 is analyzed using the pattern mining algorithm. The three highest ranked possible sources are listed in Table 1, and Figure 3 shows their MW outputs. In Figure 3, the actual MW plots of each generator are presented on the left side of each subplot. The right side of the subplots shows the values of the detrended data. The red horizontal line in the right subplot of Figure 3 depicts the $3 \sigma$ threshold for this data set.
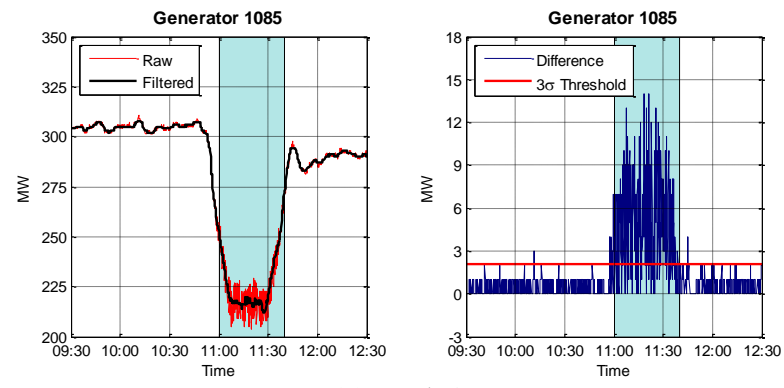

(a) Rank 1
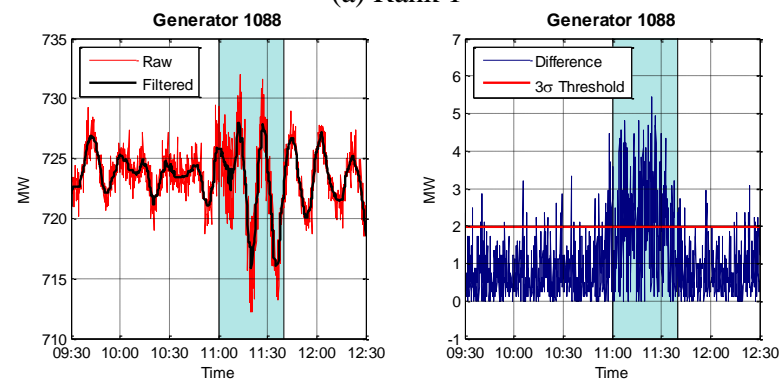

(b) Rank 2
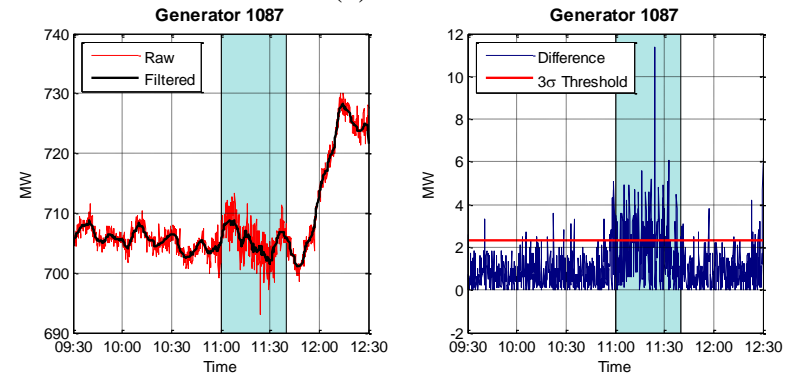

(c) Rank 3

Figure 3. MW outputs of the three highest ranked generators using PMA
The pattern mining algorithm selects generators 1085,1088 and 1087 as the potential candidates for the oscillation source according to Table 1 . Observable oscillations can be seen from all three MW outputs during the oscillation window. However, generator 1085 has a ranking index two times that of the indexes for the other two. Indeed, higher amplitude oscillation activity can be seen in generator $1085 \mathrm{MW}$ output in Figure 3(a) compared to the MW outputs in Figures 3(b) and 3(c).

\subsection{Maximal Variance Ratio Algorithm}

The same data sanity check as in the pattern mining algorithm is first applied. The data is then detrended using a third order band pass filter. For the $0.1 \mathrm{~Hz}$ sampling rate (10 second SCADA update rate), the corner frequencies are set to be $0.005 \mathrm{~Hz}$ and $0.035 \mathrm{~Hz}$ for the bandpass filter. Then, the MW (or MVAR depending on the nature of the oscillation) output of the generator causing the oscillation is expected to show sustained oscillation (like in Figure 1) with the highest "amplitude" among all such signals.

Two key factors are considered when calculating the ranking index $K_{M V R A}$ in this approach. One is the number of times the data values cross their mean value within the oscillation window $N_{o s c}$, which indicates how much the MW data is showing sustained oscillations. The other one is the average standard deviation of the SCADA signal, which is a measure of the oscillation amplitude.

In order to accommodate the slow sampling rate of the SCADA data, we suggest estimating the oscillation amplitude by taking an average of standard deviations from multiple moving windows. That is, let us first compute the standard deviation $\sigma_{1}$ of a defined analysis window, say 30 samples (5 minutes). And then move the analysis window along the time axis with a fix step, say 6 samples (1 minute). Next, calculate the standard deviation $\sigma_{2}$ of the new window. Keep moving the analysis window and computing the standard deviation $\sigma_{i}(i=3,4, \ldots)$ until the end of the data.

The initial ambient window is set to be the first 20 minutes of the data set (9:30 AM to 9:50 AM). The moving standard deviations are calculated over both the initial ambient window and the oscillation window, and the averages of the moving standard deviations for the two windows are denoted as $S T D_{a m b}$ and $S T D_{o s c}$, respectively.

This moving window approach can be used to extend the algorithm towards online implementation in the future. $S T D_{a m b}$ can easily be estimated in online framework from routine ambient SCADA data that is available all the time. Then, once a sustained oscillation is detected by a PMU based oscillation 
detection algorithm such as FFDD in [8], if the oscillations persist long enough (say longer than 5 minutes), the corresponding SCADA data during the oscillation time period can be used to estimate $S T D_{o s c}$ from SCADA data.

The ranking index for each signal is defined as

$$
K_{{M V R A_{-} i}}=N_{\text {osc }_{-} i} \frac{S T D_{\text {osc }_{-} i}}{S T D_{a m b_{-} i}}, \quad i=1,2, \ldots, n .
$$
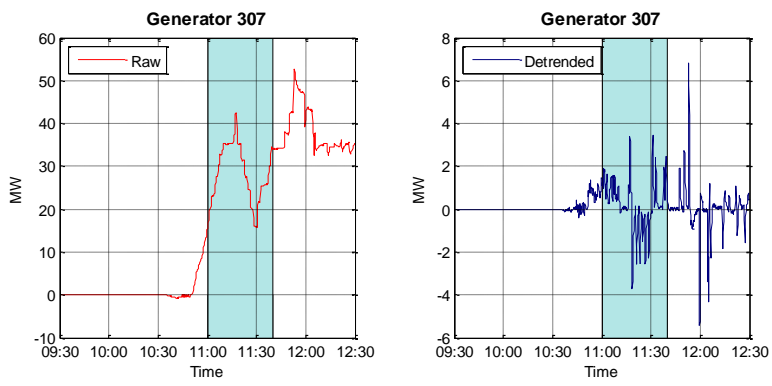

(a) Filter 1
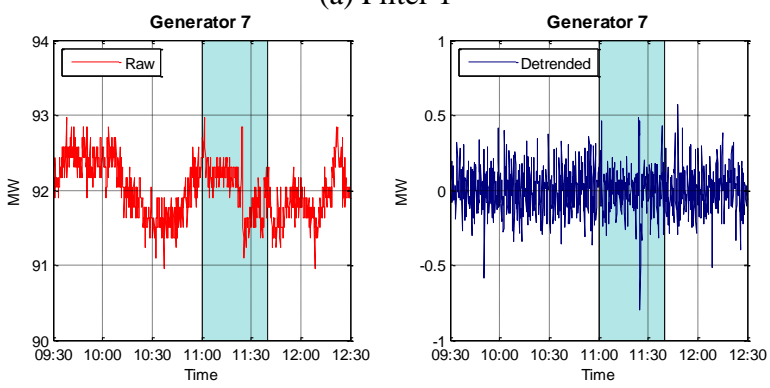

(b) Filter 2
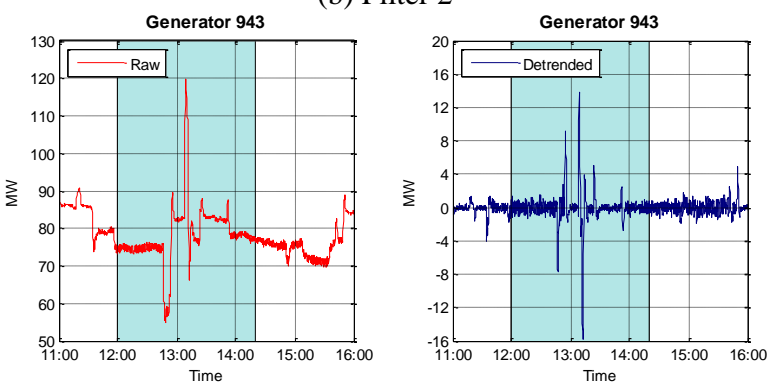

(c) Filter 3
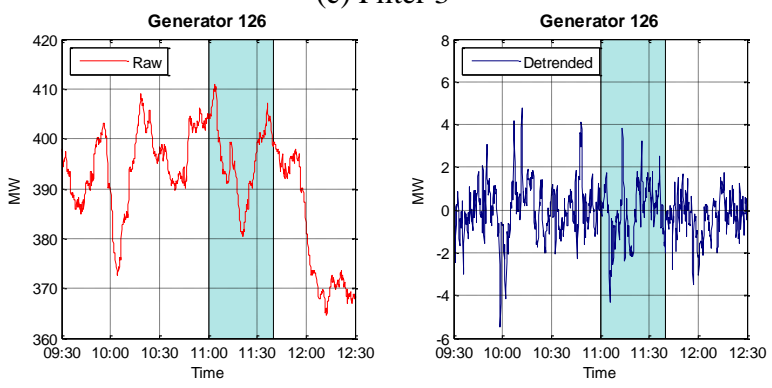

(d) Filter 4

Figure 4. Example of signals ruled out by different filters in MVRA
It is noted that, $K_{M V R A_{-} i}$ will become very large when $S T D_{a m b \_} i$ is too small. This can happen for the generators which were off during the window and for SCADA signals with low resolution or low sensitivity. Such signals that remained mostly unchanged during the initial ambient window will be excluded with Filter 1. For example, Figure 4(a) shows a generator MW output whose $S T D_{a m b_{-} i}$ is zero, so that its $K_{M V R A_{-} i}$ in (2) is infinity.

The second check is for the moving variance $S T D_{\text {osc }_{-} i}$ of the signal during the oscillation window. Low value of $S T D_{\text {osc } i}$ suggests there was mainly ambient activity (not oscillations) in the MW data during the oscillation window. Such generators are not candidates to be oscillation sources and can be omitted from further analysis. An example of such a signal is shown in Figure 4(b), which will be ruled out by Filter 2.

As a next example, the signal in Figure 4(c) has a

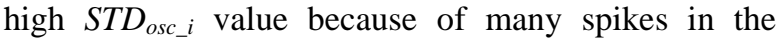
oscillation window even though the oscillation activity amplitude is relatively small. In our experience, forced oscillations tend to be sustained with high amplitude over the entire oscillation window (like in Figure 3(a)) for the potential oscillation sources. Therefore, generator outputs like in Figure 4(c) with many spikes will be ruled out from the analysis with Filter 4. This example in Figure 4(c) is from another oscillation event which will be introduced in Section 4.

Filter 4 rejects the channel whose crossover number $N_{o s c}$ is too small. For example, the generator output in Figure 4(d) ramped up and down during the oscillation window. Because of the MW ramp, it has a high moving standard deviation $S T D_{\text {osc }_{-} i}$. However, the generator can also be excluded because it did not show much oscillation during the time window of interest.

Detrending using a bandpass filter noted in the beginning of Section 3.2, and a clipping limiter have been applied in order to remove the slow trends of the signals and to reduce the effect of sudden data spikes, respectively.

The main steps of the maximal variance ratio algorithm are summarized below.

1) Input SCADA data of generators and the oscillation event time from FFDD analysis of PMU data.

2) Data sanity check.

3) Calculate the average of the moving standard deviations for the initial ambient window. Reject the channel if the maximal difference of the data during the window is less than a preset multiple of the average standard deviation (Filter 1).

4) Calculate the average of the moving standard deviations for the oscillation window. Reject the channel if this average standard deviation is less than the minimum oscillation threshold (Filter 2). 
5) Detrend using the bandpass filter.

6) Reject the channel if the number of spikes inside the oscillation window is no less than the spike count threshold (Filter 3).

7) Apply the clipping limiter for the oscillation window.

8) Count the number of times the data values cross their mean value within the oscillation window $N_{\text {osc } \_ \text {i. }}$.

9) Reject the channel if $N_{o s c_{-} i}$ is less than a preset factor of the number of samples inside the oscillation window (Filter 4).

10) Recalculate the moving standard deviations over both the initial ambient window and the oscillation window for the filtered data, and compute the average $S T D_{a m b \_} i$ and $S T D_{\text {osc } \_}$for the two windows, respectively.

11) Compute the ranking index $K_{M V R A} i$ according to (2).

12) Apply step 2 to 11 for the rest of channels.

13) Select Top 3 channels based on the ranking index.

14) Inspect the MW outputs of the possible oscillation sources for manual verification.

The maximal variance ratio algorithm is applied to Event 1 in Section 2. Three generators with the largest ranking index values are listed in Table 2 and their MW outputs are plotted in Figure 5.

According to Tables 1 and 2, same three generators have been selected by both methods, and the channel generator 1085 stands out from the rest of the channels.

\subsection{Validation}

The results from two methods mostly agree with each other. And, generator 1085 is located in the area where the oscillation shape results in Figure 2(b) pointed to. In fact, the PMU channel with the largest magnitude in oscillation shapes is the one closest to generator 1085.

The findings have been verified by discussion with the owner of the generation station. A mechanical failure occurred on the particular generation unit we identified, which caused the forced oscillation in the system. The second and third ranked generators 1087 and 1088 are two other units in the same generation plant and they were responding to the forced oscillation in unit 1085 . Therefore, they likely had the next highest amplitudes after unit 1085. In conclusion, the ranking by the two methods PMA and MVRA have correctly identified the source of the forced oscillation from over 2000 generators in the system using SCADA data.
Table 2. Possible sources identified using MVRA

\begin{tabular}{|c|l|c|c|c|c|}
\hline Ranking & Channel Name & $\begin{array}{c}\text { Ranking } \\
\text { Index } \\
\boldsymbol{K}_{\boldsymbol{M V R A} \boldsymbol{A}}\end{array}$ & $\boldsymbol{S T D}_{\text {amb }}$ & $\boldsymbol{S T D}_{\text {osc }}$ & $\boldsymbol{N}_{\text {osc }}$ \\
\hline 1 & Generator 1085 & 777.2 & 0.5643 & 4.8736 & 90 \\
\hline 2 & Generator 1087 & 312.7 & 0.7603 & 2.1810 & 109 \\
\hline 3 & Generator 1088 & 291.1 & 0.7410 & 2.0943 & 103 \\
\hline
\end{tabular}
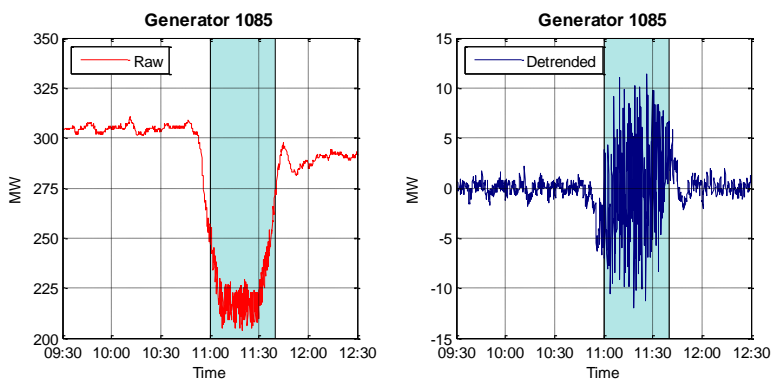

(a) Rank 1
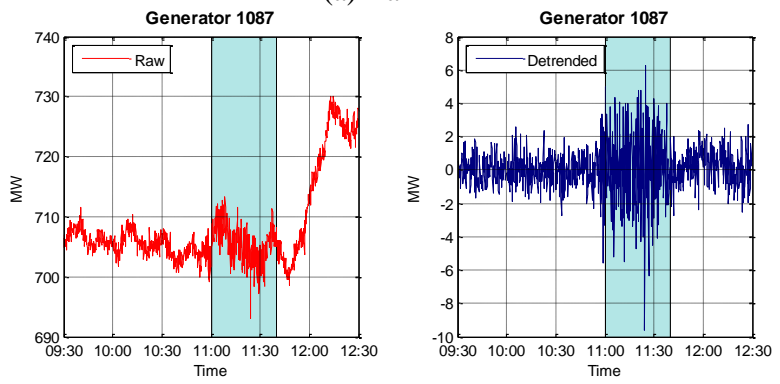

(b) Rank 2
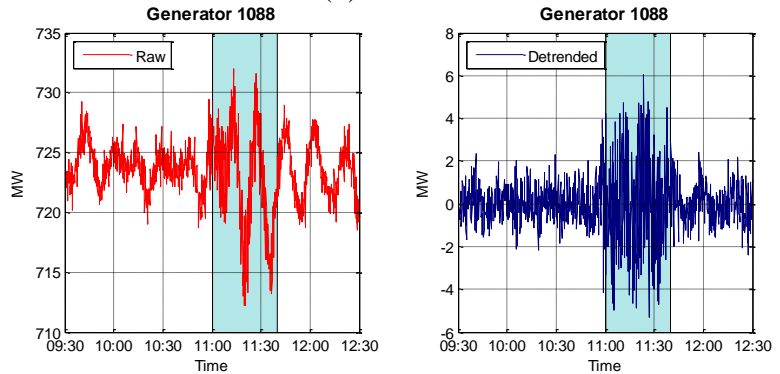

(c) Rank 3

Figure 5. MW outputs of the three highest ranked generators using MVRA

\section{Other oscillation events}

The proposed methods have been applied to two other oscillation events found in western interconnection power system for validation.

\subsection{Event 2 on January 28, 2015}

The same oscillation discussed in Event 1 on the next day which serves as the second validation case. Estimation results of PMU data using FFDD [8] are provided in Figure 6. The oscillation returned from 
around 12:00 PM to 2:20 PM with an oscillation frequency at $1.11 \mathrm{~Hz}$ and the damping ratio was again estimated to be very low at $1.0 \%$. The oscillation shape shown in Figure 6(b) was similar to that of Figure 2(b) and it pointed to the same area of the system as in Section 3. The SCADA data from 11 AM to 4 PM was pulled from the historian, and the two methods proposed in Section 3 are applied.

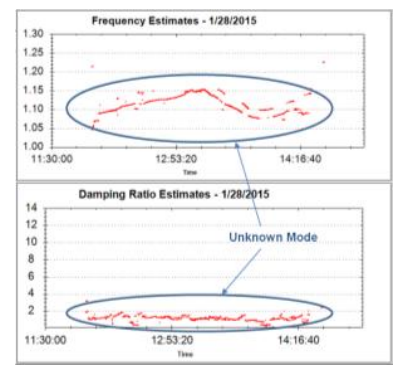

(a) Summary plot

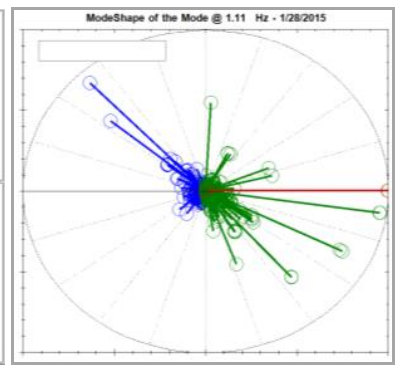

(b) Oscillation shape
Figure 6. Detection of the second forced oscillation using FFDD
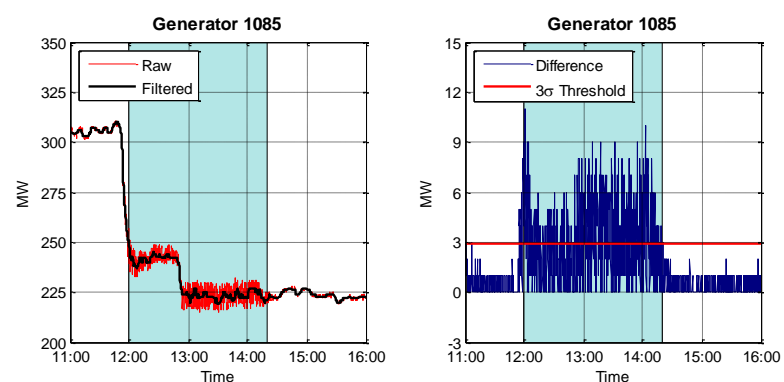

(a) Rank 1
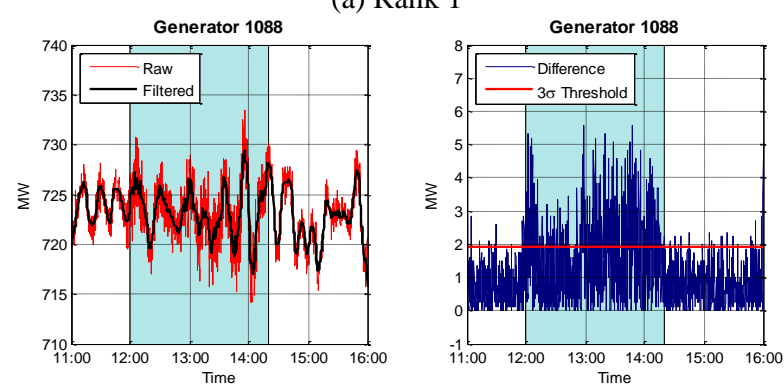

(b) Rank 2
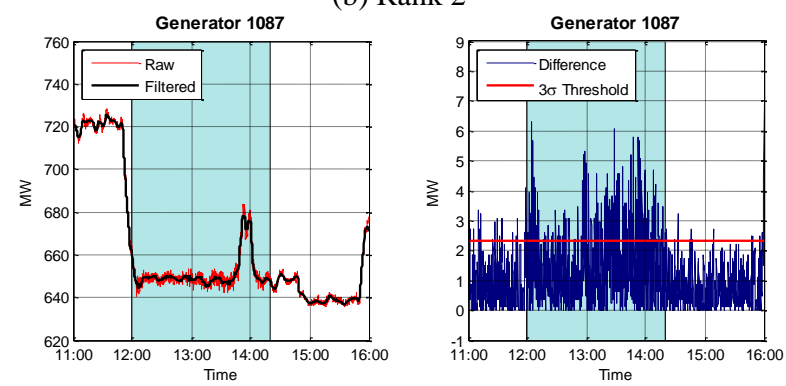

(c) Rank 3

Figure 7. MW outputs of the three highest ranked generators using PMA
Tables 3 and 4 summarize three possible oscillation sources located using PMA and MVRA, respectively. Their generation outputs are plotted in Figure 7 and Figure 8.

Table 3. Possible sources identified using PMA Length $_{\text {osc }}=841$, Length Lmb $=960$.

\begin{tabular}{|c|c|c|c|c|}
\hline Ranking & Channel Name & $\begin{array}{c}\text { Ranking } \\
\text { Index } \boldsymbol{K}_{\boldsymbol{P M A}}\end{array}$ & NUM $\boldsymbol{M s c}_{\boldsymbol{s}}$ & NUMamb \\
\hline 1 & Generator 1085 & 0.1888 & 164 & 6 \\
\hline 2 & Generator 1088 & 0.1082 & 112 & 24 \\
\hline 3 & Generator 1087 & 0.0619 & 74 & 25 \\
\hline
\end{tabular}

Table 4. Possible sources identified using MVRA

\begin{tabular}{|c|c|c|c|c|c|}
\hline Ranking & Channel Name & $\begin{array}{c}\text { Ranking } \\
\text { Index } \\
\text { KMVRA }_{\text {MV }}\end{array}$ & STD $_{\text {amb }}$ & STD $_{\text {osc }}$ & $\boldsymbol{N}_{\text {osc }}$ \\
\hline 1 & Generator 1085 & 1498.4 & 0.6422 & 2.9161 & 330 \\
\hline 2 & Generator 1087 & 617.9 & 0.7699 & 1.5909 & 299 \\
\hline 3 & Generator 1088 & 414.6 & 1.1204 & 1.6240 & 286 \\
\hline
\end{tabular}
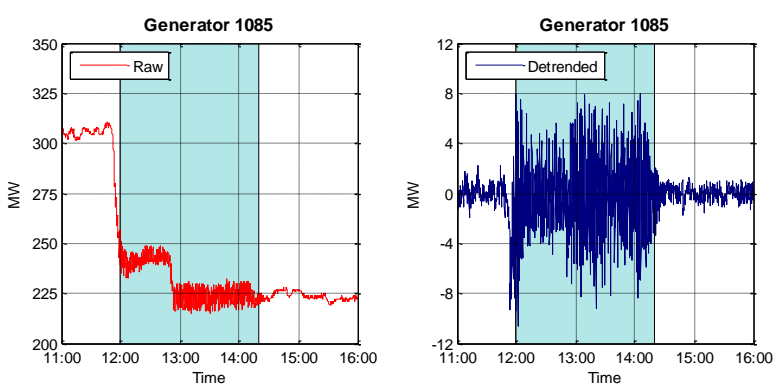

(a) Rank 1
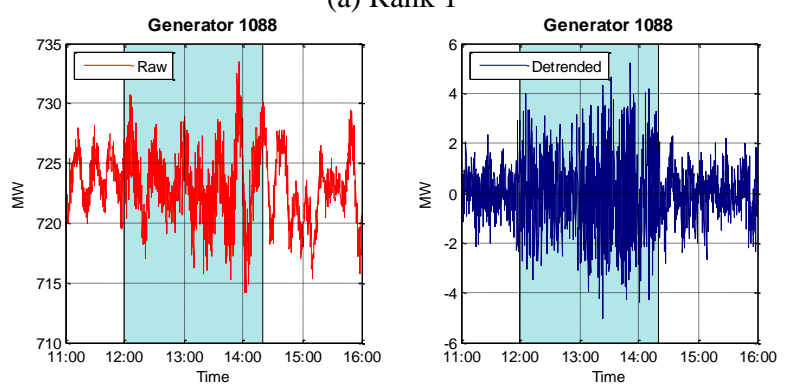

(b) Rank 2
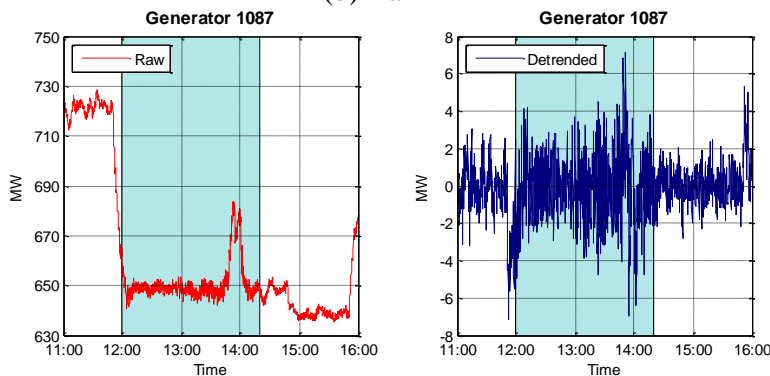

(c) Rank 3

Figure 8. MW outputs of the three highest ranked generators using MVRA 
Like in the case of Event 1, generator 1085 is ranked the most likely candidate to be the source of the forced oscillation for Event 2 as well. Again, two other units in the same plant, namely, generators 1087 and 1088 are ranked the next highest by both methods. As stated earlier, a mechanical valve failure in generator 1085 did indeed cause the forced oscillation for this event as well and the source of the oscillation being generator 1085 was confirmed by the generation owner.

\subsection{Event 3 on March 10, 2015}

The third oscillation event was detected on March 10,2015 , which propagated through a major portion of the western interconnection power system.

Figure 9 provides the estimation results of the FFDD engine from [8]. It shows that an oscillation was detected from around 11:02 AM to 11:07 AM. The oscillation frequency was at $1.47 \mathrm{~Hz}$ and the damping ratio was estimated to be very low as $0.34 \%$, which indicated that a possible forced oscillation had occurred. In this event, since there was no PMU close to the oscillation source, there were dozens of PMU channels whose magnitudes were relatively large in the oscillation shape results in Figure 9(b). The oscillation can be clearly seen in the time plot of a line current magnitude from a PMU shown in Figure 9(c). Compared to the two previous oscillation events discussed earlier in the paper, this case is more challenging because the oscillations lasted only about 5 minutes. The 5 minute oscillation window consists of only 30 SCADA data points at the 10 second sampling rate.

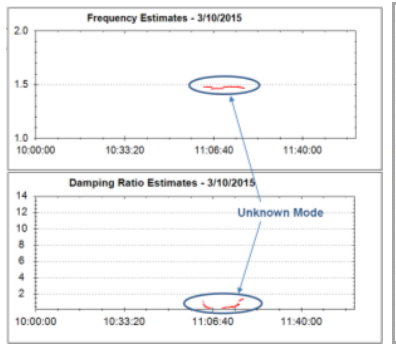

(a) Summary plot

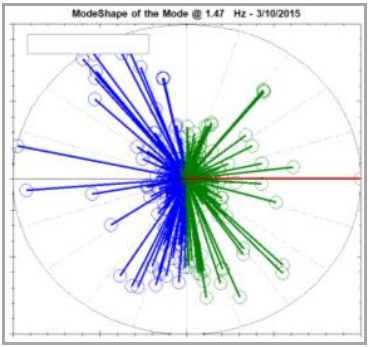

(b) Oscillation shape

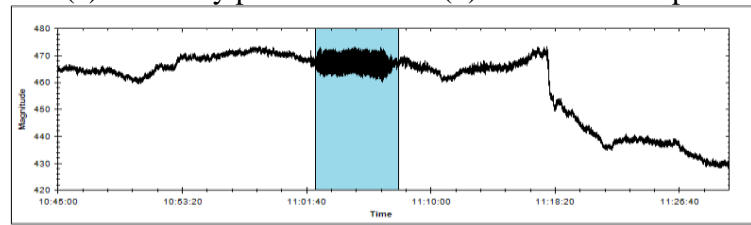

(c) Time-plot of a line current magnitude from a PMU

Figure 9. Detection of the third forced oscillation event using FFDD

To apply the two algorithms of Section 3, SCADA data from $10 \mathrm{AM}$ to $12 \mathrm{PM}$ was extracted from the historian and the ranking indices of Section 3 were estimated. The three highest ranked possible oscillation sources identified using PMA and MVRA are summarized in Table 5 and Table 6, respectively. Their SCADA generation outputs are shown in Figures 10 and 11.

Table 5. Possible sources identified using PMA Length $_{\text {osc }}=31$, Length Lmb $_{\text {. }}=690$.

\begin{tabular}{|c|c|c|c|c|}
\hline Ranking & Channel Name & $\begin{array}{c}\text { Ranking } \\
\text { Index KPMA }\end{array}$ & NUM $\boldsymbol{s s c}_{\text {s }}$ & NUM amb \\
\hline 1 & Generator 1215 & 0.2903 & 9 & 0 \\
\hline 2 & Generator 2278 & 0.2186 & 7 & 5 \\
\hline 3 & Generator 945 & 0.1410 & 5 & 14 \\
\hline
\end{tabular}

Table 6. Possible sources identified using MVRA

\begin{tabular}{|c|c|c|c|c|c|}
\hline Ranking & Channel Name & $\begin{array}{c}\text { Ranking } \\
\text { Index } \\
\boldsymbol{K}_{\text {MVRA }}\end{array}$ & $\boldsymbol{S T D}_{\text {amb }}$ & $\boldsymbol{S T D}_{\text {osc }}$ & $\boldsymbol{N}_{\text {osc }}$ \\
\hline 1 & Generator 1215 & 1031.1 & 0.1142 & 9.8159 & 12 \\
\hline 2 & Generator 2278 & 113.1 & 0.1397 & 1.5795 & 10 \\
\hline 3 & Generator 2280 & 56.3 & 0.7653 & 3.9141 & 11 \\
\hline
\end{tabular}
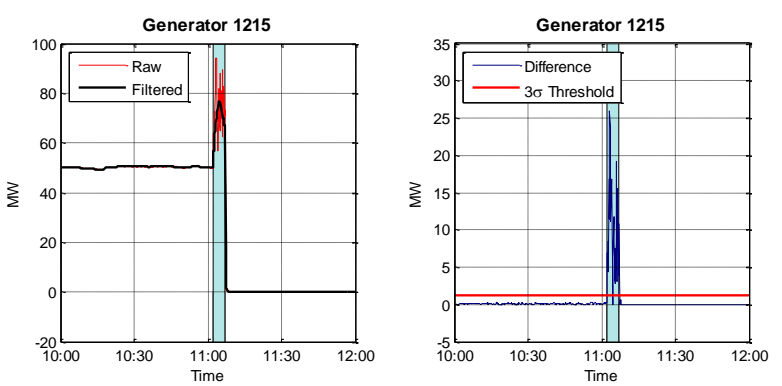

(a) Rank 1
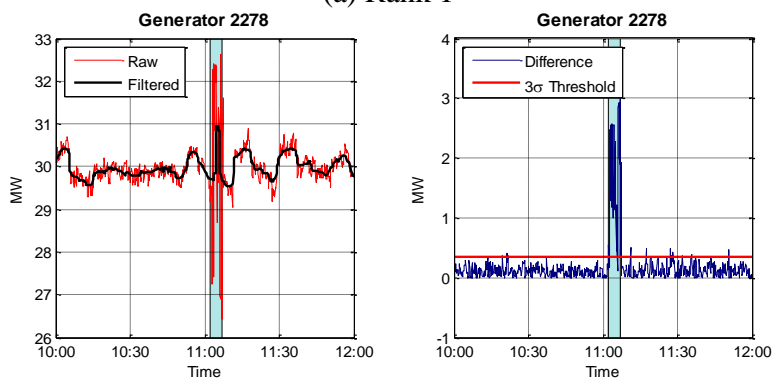

(b) Rank 2
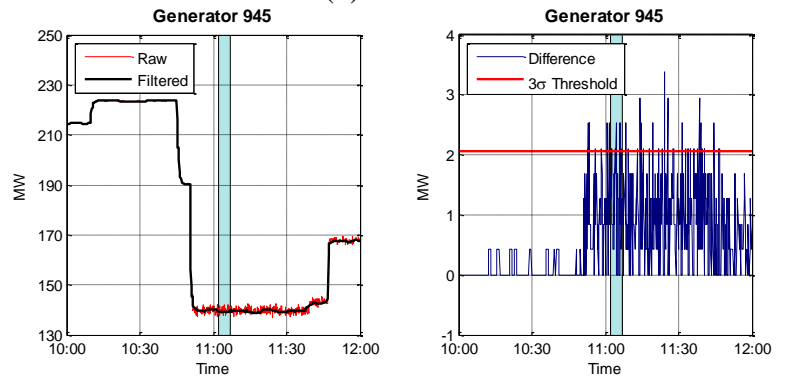

(c) Rank 3

Figure 10. MW outputs of the three highest ranked generators using PMA 
Generator 1215 has been identified as the most likely cause of the forced oscillation by both methods. The generator owner has confirmed that a control problem occurred during the time period of the forced oscillation which validates the findings from the two proposed methods. It appears that the third ranked generators in both methods look a little suspect and they may be unrelated to the oscillation event at generator 1215. However, both methods are able to correctly identify the oscillation source, generator 1215 in Figures 10(a) and 11(a). They also correctly point to the next highest ranked unit, namely generator 2278, which is clearly reacting to the same oscillation event as shown in Figures 10(b) and 11(b).
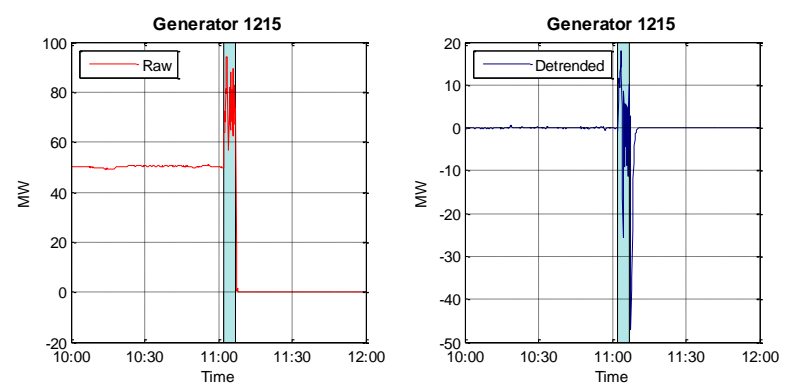

(a) Rank 1
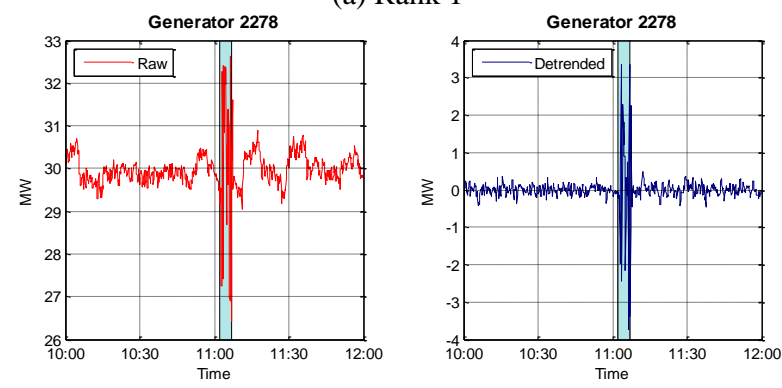

(b) Rank 2
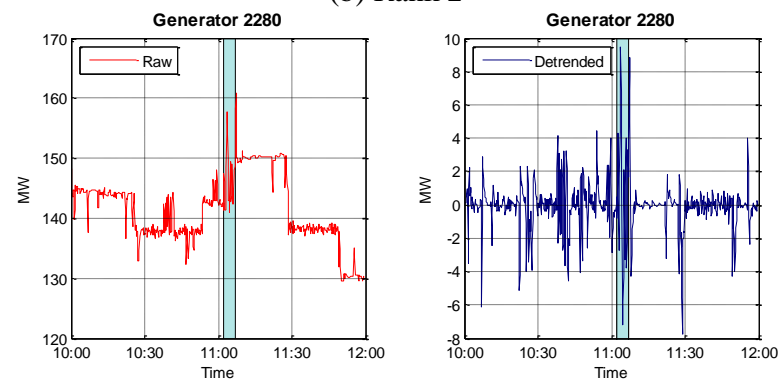

(c) Rank 3

Figure 11. MW outputs of the three highest ranked generators using MVRA

Table 7. Computational times (seconds)

\begin{tabular}{|c|c|c|c|}
\hline Event No. & 1 & 2 & 3 \\
\hline PMA & 1.6845 & 2.8922 & 1.2313 \\
\hline MVRA & 1.7032 & 3.8294 & 0.8529 \\
\hline
\end{tabular}

\subsection{Computational times}

The computational times for source locations of all three events using both methods are summarized in Table VII. They are reported from tests using Matlab 8.1 on a workstation with Intel Xeon CPU E5-2650 v2 @ $2.60 \mathrm{GHz}$ and Windows 7 operating system. All the times in Table 7 are well below the SCADA update rate of 10 seconds which indicates that the two methods can be extended for online implementation in the future.

\section{Conclusion}

PMU-based oscillation detection tools can detect occurrence of forced oscillations in power systems. They can provide a clear indication of the time window when the oscillations were present and related information such as frequency, damping ratio, and the oscillation shape. Although oscillation shape results can point to a specific area of the system that might cause the problem, they are often not conclusive enough. Further investigation is needed in order to locate the particular source of the oscillations.

In this paper, SCADA data is used for automatic source location of forced oscillations for the first time. It is not straightforward to use SCADA data by itself to detect the oscillations due to its slow sampling rate and high noise level. However, this paper shows that SCADA data becomes extremely useful for source location when combined with oscillation monitoring results from PMU data.

Two methods have been proposed for identifying oscillation sources using SCADA data automatically. It is shown that about 5 minutes of oscillation data are sufficient for ranking potential oscillation sources in both methods. The process has been used successfully through the identification of several forced oscillations that might have gone undetected without an exhaustive manual data search. What used to take an experienced engineer many hours to do, can be performed within seconds using the proposed methods.

The high availability of SCADA data makes the methods helpful in the recognition of likely problematic generators when a forced oscillation has been detected by the PMU-based oscillation monitoring tool.

\section{Acknowledgement}

We thank US Department of Energy for supporting this research through funding from the Consortium for Electric Reliability Technology Solutions. 


\section{References}

[1] J. Van Ness, "Response of large power systems to cyclic load variations, "IEEE Trans. Power App. Syst., vol.PAS-85, no.7, pp. 723-727, Jul. 1966.

[2] C. D. Vournas, N. Krassas, and B. C. Papadias, "Analysis of forced oscillations in a multimachine power system," in Proc. Int. Conf. Contr., Edinburgh, U.K., Mar. 1991, pp. 443-448.

[3] J. Ma, P. Zhang, H. Fu, B. Bo and Z. Dong, "Application of Phasor Measurement Unit on Locating Disturbance Source for Low-Frequency Oscillation," IEEE Trans. Smart Grid, vol.1, no.3, pp. 340-346, Dec. 2010.

[4] N. Zhou, "A cross-coherence method for detecting oscillations, " IEEE Trans. Power Syst., DOI: 10.1109/TPWRS.2015.2404804, to appear.

[5] J. Follum and J. W. Pierre, "Detection of periodic forced oscillations in power systems," IEEE Trans. Power Syst., DOI: 10.1109/TPWRS.2015.2456919, to appear.

[6] S. A. Nezam Sarmadi and V. Venkatasubramanian, "Inter-area resonance in power systems from forced oscillations," IEEE Trans. Power Syst., DOI: 10.1109/TPWRS.2015.2400133, to appear.

[7] K. Kim, et al., "Methods for calculating oscillations in large power systems," IEEE Trans. Power Syst., vol. 12, no. 4, pp. 1639-1648, Nov. 1997.

[8] H. Khalilinia, L. Zhang, and V. Venkatasubramanian, "Fast frequency-domain decomposition for ambient oscillation monitoring", IEEE Trans. Power Del., vol.30, no. 3, pp. 1631-1633, Jun. 2015. 